\title{
A Multi Band EBG Resonator Antenna for Performance Enhancement in UHF Band Frequency Range
}

\author{
Gaurav Jain \\ Department of \\ Electronics and \\ Communication Engineering, \\ Sagar Institute of Research, \\ Technology and Science \\ Bhopal, India
}

\author{
Nitesh Kumar \\ Department of \\ Electronics and \\ Communication Engineering, \\ Sagar Institute of Research, \\ Technology and Science \\ Bhopal, India
}

\begin{abstract}
A multiple band electromagnetic band gap (EBG) antenna is very important discussion topic in the area of antennas design because of its numerous advantages over conventional antennas i.e low cost, simple to feed, light in weight, easy fabrication process and their effective radiation characteristics. Patch antenna is also used with Microwave IC's and Monolithic Microwave IC's because of its compatibility. Utilization of electromagnetic band-gap (EBG) structures is becoming attractive in the electromagnetic and antenna community, In this paper, the effects of a twodimensional electromagnetic bandgap (EBG) Structures on the performance of microstrip patch antenna and its overview study in past few decades are presented. Antenna gain enhancement using a holey dielectric superstrate on a microstrip patch antenna is also studiedthe main objective of this paper is to get an inside into an EBG structure in order to work it as a resonator and various techniques to improve its bandwidth and gain for multiband application.
\end{abstract}

\section{Keywords}

Electromagnetic band gap (EBG), EBG resonator antenna (ERA), Broadband and Multi band shorting pins.

\section{INTRODUCTION}

ELECTROMAGNETIC band gap (EBG) structures exhibit unique characteristics to guide, filter, or collimate electromagnetic waves and have attracted a lot of interest from the research community in recent years [9]-[10]. Particularly, their antenna applications in suppressing surface waves; reducing antenna profile [12], [15]; as well as forming highly directive antennas have been of considerable interest [12][15]. EBG resonator antennas (ERAs), also known as FabryPerot cavity antennas, are well known for their ability to increase broadside directivity of simple antennas without resorting to complex feed networks as in phase arrays. Although pioneering research on this concept was published by Von Trentini in 1956 [9], Jacksonet al. were the first to propose the use of unprinted dielectric slabs as superstrates for gain enhancement in 1985 [3], [11]. The recent surge of research in EBGs and periodic structures inspired many researchers to revisit this concept and make significant advances. A common EBG resonator antenna (ERA) consists of an air-filled half-wavelength cavity formed between a perfect reflector (e.g., metallic ground plane or AMC) and a high-reflectivity top. This top section can take various forms, depending upon the design methodology and fabrication technology, including 3-D EBG structures, in this paper we are using 2-D printed frequency-selective surfaces and stacks of unprinted dielectric slabs [4]-[5]. Here, we use the general term superstructure to refer to all such top sections. A variety of superstructures has been investigated. Their size reduction [6], [11]; bandwidth

enhancement [11]-[16], have been addressed for applications, such as mobile base stations, global satellite coverage, feed clusters for multiple-beam antennas in space applications, and point-to-point microwave links in frequency range of 1 to $5 \mathrm{GHz}$.

This paper studies the single dielectric superstrate to a microstrip antenna for increasing antenna gain. By applying the superstrate to the microstrip antenna, a wider application such as radome and housing can be possible. To create an inphase radiation aperture, the effective permittivity of the superstrate at each position is controlled by drilling holes in the dielectric layer. In section II, the effective permittivity and mutual coupling of the superstrate is studied. In section III, survey of the Development of EBG Structures in antenna is presented. In section IV, the simulation and measured results of various cases are compared. And finally, a conclusion is reached in section $\mathrm{V}$.

\section{The EFFECTIVE PERMITTIVITY AND MUTUAL COUPLING OF THE SUPERSTATE}

A. In-phase calculations on different effective permittivity Superstrates

The electric field distribution of the antenna aperture and the radiation pattern have a Fourier transform relationship. Therefore in order to create a high gain antenna, it is not only necessary to have a larger antenna aperture, but there should also be an in-phase electric field on the aperture [1]. Let us assume a point source as shown in Fig. 1. The phase of the electric field some distance away from the point source is dependent on the distance. The phase difference $(\Delta \xi)$ between observations points $\mathrm{P} 1$ and $\mathrm{P} 2$ in the air can be expressed as following equation.

$$
2 n \pi+\Delta \xi=\boldsymbol{\beta}_{0}\left(\sqrt{\boldsymbol{d}^{2}+\boldsymbol{l}^{2}}-d\right)
$$

Where $\boldsymbol{\beta}_{\mathbf{0}}$ is the propagation constant in the air, $\boldsymbol{d}$ is the distance between the point source and P1, $\boldsymbol{l}$ is the distance between P1and P2. According to (1), the phase difference is always nonzero when the distance $\boldsymbol{l}$ is not zero. When a dielectric layer which has a thickness of $\boldsymbol{d} \mathbf{2}$ with a different permittivity determined by its position is located above the point source at a distance of $\boldsymbol{d} 1$, the phase difference $(\Delta \xi)$ between points $\mathrm{P} 1$ and $\mathrm{P} 2$ can be expressed by the following equation, assuming that there is no reflection between the dielectric layer and the air: 
$2 n \pi+\Delta \xi=\boldsymbol{\beta}_{\mathbf{0}}\left(\sqrt{\boldsymbol{d}^{2}+\boldsymbol{l}^{\mathbf{2}}}-\boldsymbol{d}_{\mathbf{1}}\right)+\boldsymbol{d}_{\mathbf{2}} \boldsymbol{\beta}_{\mathbf{0}}\left(\sqrt{\boldsymbol{\varepsilon}_{\mathbf{1}}}-\sqrt{\boldsymbol{\varepsilon}_{\mathbf{2}}}\right)$ Where $\varepsilon_{2}$ is the permittivity at $\mathrm{P} 2$ and $\varepsilon_{1}$ is permittivity at $\mathrm{P} 1$. According to (2) the phase difference $\Delta \xi$ can be set to zero by $\varepsilon_{2}$.

The effective permittivity can be controlled by changing the properties of the material, such as its thickness. In this paper, creating holes in the dielectric layer is suggested in order to obtain different effective permittivities according to the size of the holes. CST MWS, a 3D FDTD based EM simulation, was used to choose the proper hole size. Fig. 2(a) shows a unit cell simulation structure. PEC and PMC boundary conditions are used. Since multiple reflections occur at the surface of the dielectric layer it was possible to obtain the relationship between hole size and effective permittivity using a unit cell simulation.

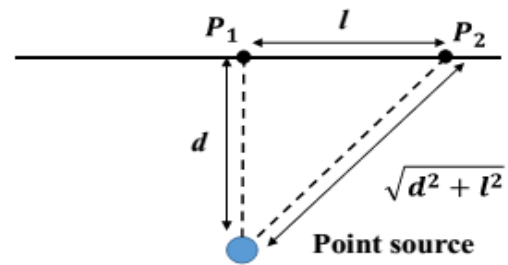

(a)

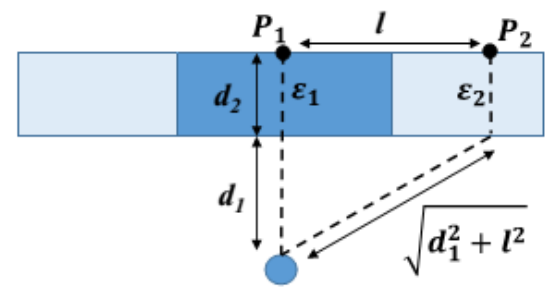

(b)

Fig. 1. Phase difference between two points for the air (a) and effective superstrate (b)



(a)

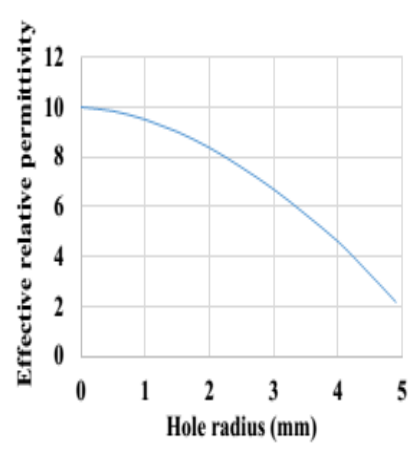

(b)
Fig. 2. Unit cell simulation model (a) and extracted effective permittivity for the different hole radius (b)

It is observed that the value of the effective permittivity is inversely proportional to the radius of the holes [1]. With respect to holes with a radius of $1,2,3$, and $4 \mathrm{~mm}$, the values of effective permittivity are calculated as 9.5, 8.3, 6.5, and 4.5

B. Techniques to reduce mutual coupling

Mutually coupling among closely spaced radiating elements is essential for the performance of microstrip antenna systems due to fact that induced currents, input impedance and radiation patterns are affected when antenna elements are correlated which in

turn reduces capacity of MIMO system [17]. Simple solution to reduce correlation is through physically separating radiating elements by distance greater than $\lambda / 2$. Basically Electromagnetic band gap (EBG) structures are realized by periodic arrangement of dielectric materials and shows characteristics of band-pass or bandstop [5] and makes isolation between components [6]. Coupling between the microstrip antenna arrays is the essential problem that always exists. To solve the problem, metamaterial is used in a rectangular patch antenna array substrate in order to reduce coupling between array antennas [7]-[9].

EBG structure has strong effect in reducing the surface wave in E-plane condition; hence the coupling between antennas will be reduced. A mushroom-like EBG structure is implemented in the design of microstrip antenna arrays to reduce the strong mutual coupling caused by the thick and high permittivity substrate without sacrificing the compact size or bandwidth of the antenna elements. The EBG structure is analyzed using the FDTD method. Compared to other approaches such as cavity back structure, the EBG structure demonstrates a better performance to improve the mutual coupling. A novel structure based on complementary splitring resonators (SRRs) is introduced [15] to reduce the mutual coupling between two coplanar microstrip antennas which radiates in the same frequency band. The new unit cell consists of two complementary SRR inclusions connected by an additional slot. it improves the rejection response in terms of bandwidth and suppression. The SCSRRs reduce the mutual coupling between two coplanar microstrip patch antennas spaced by a quarter free-space wavelength. The reduction is possible because of the ability of the SCSRRs to efficiently suppress the electric fields normal to the ground plane, which in turn reduces the surface currents in the terminated antenna element.

A low mutual coupling design for two and four elements microstrip antenna array were proposed [17]. A new distribution for dumbbell shaped defect on the ground plane of the antenna is inserted between the patches creating a band gap in the operation frequency band of the antenna. By suppressing the surface waves, it provides a very low mutual coupling between array elements. The analysis indicates that increasing number of dumbbells reduces the mutual coupling between elements. Radiation patterns have minimal change in the broadside direction but back lobe level is increased. However, the gain and the efficiency are decreased due to penetration of DGS in the ground plane.

\section{DEVELOPMENT OF EBG STRUCTURE IN MICROSTRIP ANTENNA}

EBG structures can be broadly categorized into three groups according to their geometric configuration: (1) three dimensional volumetric structures, (2) two-dimensional planar surfaces, and (3) one-dimensional transmission lines [4]. The dimensionality depends on the periodicity directions. The first attempts towards three-dimensional structures were realized in the form of face centered cubic

(FCC) lattice structures [11]. At the initial stages of EBG research, due to the lack of theoretical predictions, a "cut and 
try" approach was adopted in experimentally predicting the band gap. Figure 3(a) shows a representative 3-D EBG structure $[16,11]$. In the late1990s ,two important planar EBG structures were invented, where metallic components were effectively incorporated into the unit cells. One is the mushroom-like EBG surface and the other is the uniplanar EBG surface which areas shown in Figure 3(b). The mushroom-like EBG exhibits band gap at lower frequencies compared to uniplanar EBG, but it is associated with fabrication complexities due to the vertical via placement and requires thick dielectric material. On the other side, uniplanar EBG has no vertical vias and is suitable for thin substrate material which makes the fabrication easier [13]. In addition, it is less sensitive to the incident angle and polarization [4]. An example of 1-D EBG transmission line design is plotted in Figure 3(c). The band gap feature of EBG structures has found useful applications in suppressing the surface waves in microstrip antenna design. These structures are also found to be effective for bandwidth improvement [11], compact microstrip antenna designs [11], harmonic control , and the radiation pattern control.

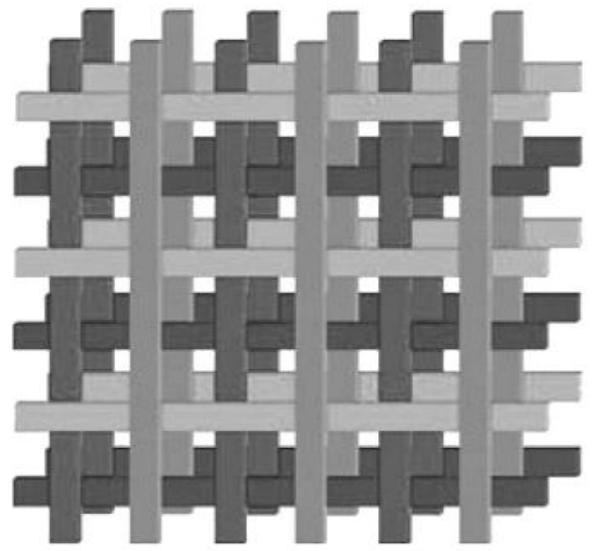

(a)

Figure 3: EBG structures: (a) 3-D EBG: a woodpile dielectric structure

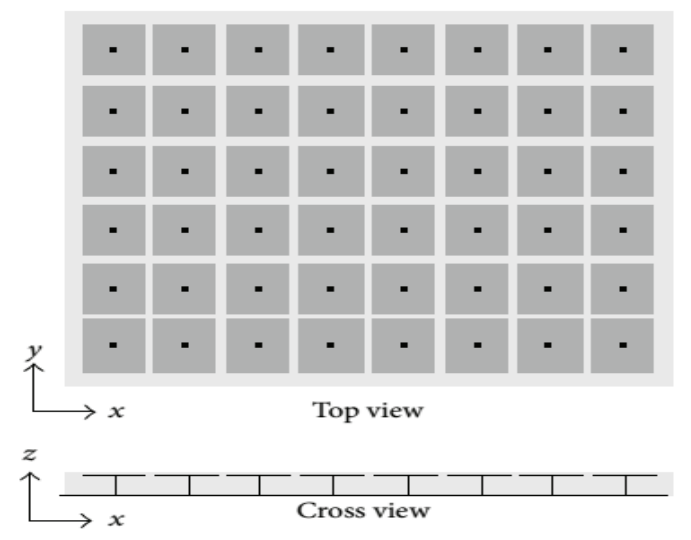

(b)

Figure 3: EBG structures: (b) 2-D EBG: a mushroom EBG

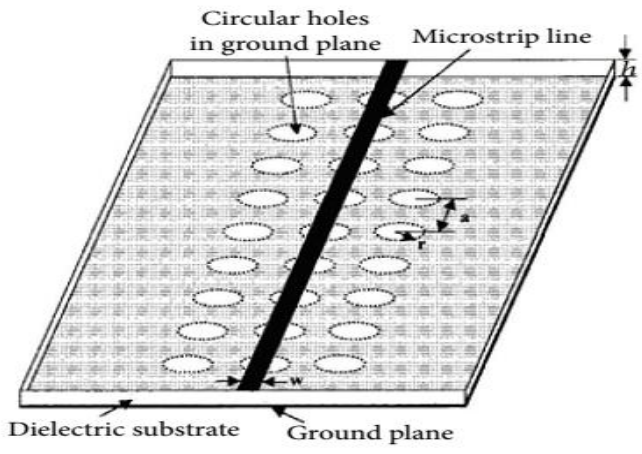

(c)

Figure 3: EBG structures (c) 1-D EBG: a microstrip line with periodic holes on the ground plane.

EBG structure is also an attractive solution to reduce the power loss through the substrate of a conventional antenna [4]. Generally, EBG structure produces a smoother radiation profile, lower back radiation, and higher gain and efficiency than a conventional ground antenna. Moreover, these structures would ensure low interference with adjacent microwave elements and act as a shielding material between the antenna and the user of communications device [5]. Furthermore, an EBG object could drive the microstrip antenna to a dual frequency operation modifying the higher order radiation patterns, thus making them similar to those of the basic mode [4].

\section{Simulation and measured results of various cases.}

\section{SIMULATION AND MEASURED RESULT OF VARIOUS CASES}

S11 and radiation patterns are discussed by comparing the microstrip patch antenna with the proposed antenna which has the holey superstrate. The simulated and measured values for S11 of both the patch antenna with the superstrate and without the superstrate are shown in Fig. 4.

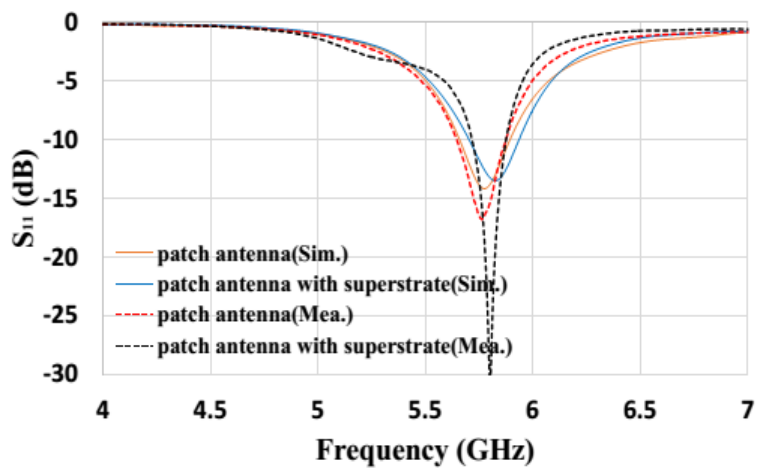

Fig. 4. Simulated and measured S11 for the patch antenna with and without the superstrate.

The simulated and measured radiation patterns of both a normal patch antenna and the proposed antenna at $5.75 \mathrm{GHz}$ are illustrated in Fig. 5. The maximum gain of the simulated and measured patterns for the single patch antenna are $4.6 \mathrm{~dB}$ and $4.5 \mathrm{~dB}$, respectively. The maximum gains of the simulated and measured patterns for the antenna including the superstrate are $6.9 \mathrm{~dB}$ and $6.8 \mathrm{~dB}$, respectively. The simulated and measured radiation patterns are in good agreement and a gain enhancement of $2.3 \mathrm{~dB}$ is observed for the measured pattern. 


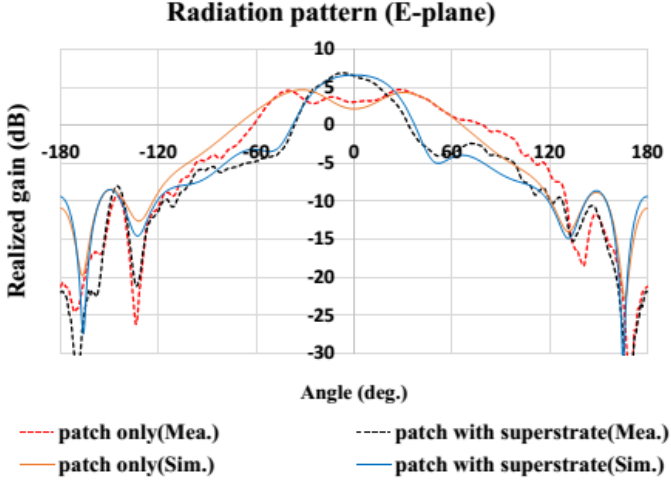

(a)



(b)

Fig. 5. Radiation patterns for the patch antenna with and without the superstrate. E-plane (a), H-plane (b)

\section{CONCLUSION}

In this paper, a comprehensive survey is presented on the

electromagnetic band gap structure, their various configurations, and successive improvement up to today's uni-planar design. For the RF and microwave researchers, the EBG terminology is a hot research direction to solve out many problems that degrade the functional efficiency of a system. An antenna is an indispensable part of a communication system to receive and transmit electromagnetic energy. Being completely planar, microstrip antennas are very attractive and widely used for applications starting from $\mathrm{MHz}$ to $\mathrm{GHz}$ frequency range. The low gain, narrow bandwidth, and poor radiation performance has limited their applications for many purposes. Another limitation is the pronounced surface waves inside the dielectric material, which worsen their radiation characteristics and increase the mutual coupling between elements of an array. Utilization of the EBG structures in antenna design is introduced as a revolutionary development with their versatility and aptness. Among the various designs, the uniplanar type EBG configurations are very attractive, easier to fabricate, and suitable for integration with a wide range of planar devices. However, compactness is an issue in designing EBG structures at desired frequencies, since the periodicity should be a half-wavelength at the center frequency. A few EBG structures are found of having band gaps at lower frequencies but those designs were not investigated with other microwave devices. As seen in the illustrated 20 International Journal of Antennas and Propagation antenna-EBG combinations, attempts have been taken in diverse ways to improve antenna element performances and to reduce the mutual coupling of microstrip antenna array. This literature study will hopefully set a basis about the current state-of-the-art of EBG research and will be helpful for microstrip antenna designs.

\section{REFERENCES}

[1] Jae Hee Kim, Chi-Hyung Ahn, Jin-Kyu Bang, "Antenna Gain Enhancement using a Holey Superstrate", IEEE Transactions On Antennas And Propagation, Vol. 64, No. 3, March 2016.

[2] Xiaoyan Zhang, Zhaopeng Teng, Zhiqing Liu, and Bincheng Li. "A Dual Band Patch Antenna with a Pinwheel-Shaped Slots EBG Substrate". Hindawi Publishing Corporation International Journal of Antennas and Propagation Volume 2015, Article ID 815751.

[3] Raheel M. Hashmi, Basit A. Zeb. "Wideband High-Gain EBG Resonator Antennas with Small Footprints and AllDielectric Superstructures". IEEE Transactions On Antennas And Propagation, Vol. 62, No. 6, June 2014.

[4] Osama M. Haraz, "Dense Dielectric Patch Array Antenna With Improved Radiation Characteristics Using EBG Ground Structure and Dielectric Superstrate for Future 5G Cellular Networks ". IEEE. Translations Volume 2, 2014.

[5] Weiwei Xu, Junhong Wang, "A Novel Microstrip Antenna With Composite Patch Structure for Reduction of In-Band RCS ". IEEE Antennas And Wireless Propagation Letters, Vol. 14, 2015.

[6] Basit Ali Zeb Nasiha Nikolic, and Karu P. Esselle, “A High-Gain Dual Band EBG Resonator Antenna with Circular Polarization IEEE Antennas And Wireless Propagation Letters, Vol. 14, 2015.

[7] Jae-Yeong Lee, Seung-Han Kim, and Jae-Hyung Jang, "Reduction of Mutual Coupling in Planar Multiple Antenna by Using 1-D EBG and SRR Structures", IEEE Transactions On Antennas And Propagation, Vol. 63, No. 9, September 2015.

[8] Changrong Liu, "Capacitively Loaded Circularly Polarized Implantable Patch Antenna for ISM Band Biomedical Applications", IEEE Transactions On Antennas And Propagation, Vol. 62, No. 5, May 2014.

[9] Quan Wei Lin, Hang Wong, "Printed Meandering ProbeFed Circularly Polarized Patch Antenna With Wide Bandwidth IEEE Antennas And Wireless Propagation Letters, Vol. 13, 2014.

[10] Chao Sun, Huili Zheng, Lingfei Zhang, and Ying Liu, "Analysis and Design of a Novel Coupled Shorting Strip for Compact Patch Antenna with Bandwidth Enhancement", IEEE Antennas And Wireless Propagation Letters, Vol. 13, 2014.

[11] Md. Shahidul Alam, "Development of Electromagnetic Band Gap Structures in the Perspective of Microstrip Antenna Design", Hindawi Publishing Corporation International Journal of Antennas and Propagation Volume 2013, Article ID 507158.

[12] Constantine A. Balanis; "Antenna Theory, Analysis and Design”, John Wiley \& Sons Inc. $3^{\text {rd }}$ edition. 2005.

[13] K. Buell, H. Mosallaei, and K. Sarabandi, "Metamaterial insulator enabled super directive array," IEEE Trans. Antennas Propag., vol.55, no. 4, pp. 1074-1085, Apr. 2007. 
[14] Ramesh Garg and Apisak Ittipiboon "Microsrtip Antenna Design Hand Book”, Artech House, Inc. 2001.

[15] M. M. Bait-Suwailam, O. F. Siddiqui, and O. M. Ramahi, "Mutual Coupling Reduction Between Microstrip Patch Antennas Using SlottedComplementary SplitRing Resonators", IEEE Antennas And Wireless Propagation Letters, vol. 9, pp. 876-878, 2010.

[16] Y. Lee, J. Yeo, and R. Mittra, "Investigation of electromagneticbandgap (EBG) structures for antenna pattern control," in Proceedings of the IEEE International Antennas and Propagation Symposium, vol. 2, pp. 11151118, June 2003

[17] Y. Horii and M. Tsutsumi, "Wide band operation of a harmonically controlled EBG microstrip patch antenna," in Proceedings of the IEEE Antennas and Propagation
Society International Symposium, vol. 3, pp. 768-771, San Antonio, Tex, USA, June 2002.

[18] J. Y. Lee, S. H. Kim, and J. H. Jang, "Reduction of Mutual Coupling in Planar Multiple Antenna by Using 1D EBG and SRR Structures" IEEE Trans. Antennas and Propagation, vol. 63, no. 9, pp 4194-4198, Sept 2015.

[19] C. Cheype, C. Serier, M. Thevenot, T. Monediere, A. Reineix, and B.Jecko, "An electromagnetic bandgap resonator antenna," IEEE Trans. Antennas Propag., vol. 50, no. 9, pp. 1285-1290, Sep. 2002.

[20] Naizhi Wang, Qiang Liu. "Wideband Fabry-Perot Resonator Antenna With Two Complementary FSS Layers". IEEE Transactions On Antennas And Propagation, Vol. 62, No. 5, May 2014. 